\title{
Extrapolation of Explicit DIMSIMs of High Order to Solve the Ordinary Differential Equations
}

\author{
Ali J. Kadhim, Annie Gorgey* \\ Mathematics Department, Faculty of Science and Mathematics, Universiti Pendidikan Sultan Idris, \\ Tanjong Malim, Perak, Malaysia \\ Email: *annie_gorgey@fsmt.upsi.edu.my
}

How to cite this paper: Kadhim, A.J. and Gorgey, A. (2019) Extrapolation of Explicit DIMSIMs of High Order to Solve the Ordinary Differential Equations. Journal of $A p$ plied Mathematics and Physics, 7, 3022-3030.

https://doi.org/10.4236/jamp.2019.712212

Received: November 6, 2019

Accepted: December 9, 2019

Published: December 12, 2019

Copyright (C) 2019 by author(s) and Scientific Research Publishing Inc.

This work is licensed under the Creative Commons Attribution International License $(\mathrm{CC}$ BY 4.0)

http://creativecommons.org/licenses/by/4.0/

\section{Open Access}

\begin{abstract}
The purpose of this research is to investigate the efficiency of explicit diagonally implicit multi-stage integration methods with extrapolation. The author gave detailed explanation of explicit diagonally implicit multi-stage integration method and compared the base method with a technique known as extrapolation to improve the efficiency. Extrapolation for symmetric Runge-Kutta method is proven to improve the accuracy since with extrapolation the solutions exhibit asymptotic error expansion, however for General linear methods, it is not known whether extrapolation can improve the efficiency or not. Therefore this research focuses on the numerical experimental results of the explicit diagonally implicit multistage integration with and without extrapolation for solving some ordinary differential equations. The numerical results showed that the base method with extrapolation is more efficient than the method without extrapolation.
\end{abstract}

\section{Keywords}

Extrapolation Technique, General Linear Methods, Diagonally Implicit Muti-Stage Integration, Explicit Methods

\section{Introduction}

Although general linear methods (GLMs) have been introduced about 40 years ago, many researchers have not considered GLMs as practical numerical methods. The most complicated for these methods is identifying the practical methods. For this reason, it is necessary to introduce a subclass of GLMs known as diagonally implicit mutistage integration, abbreviated as (DIMSIMs) [1]. DIMSIMs have been proven to be advantageous than RK methods and linear multi-step methods. DIMSIMs also have been considered very potential for efficient implementations. 
Consider the following initial-value problems

$$
\begin{aligned}
& y^{\prime}(x)=f(y(x)), \quad x \in\left[x_{0}, X\right] \\
& y\left(x_{0}\right)=y_{0}
\end{aligned}
$$

General linear methods (GLMs) is given by

$$
\begin{aligned}
Y_{i} & =\sum_{j=1}^{s} a_{i j} h f\left(Y_{j}\right)+\sum_{j=1}^{r} u_{i j} y_{j}^{[n-1]}, i=1,2, \cdots, s, \\
y_{i}^{[n]} & =\sum_{j=1}^{s} b_{i j} h f\left(Y_{j}\right)+\sum_{j=1}^{r} v_{i j} y_{j}^{[n-1]}, i=1,2, \cdots, r .
\end{aligned}
$$

where $Y_{i}$ can be approximated as follows

$$
Y_{i}=y\left(x_{n-1}+c_{i} h\right)+O\left(h^{q+1}\right),
$$

The incoming and outgoing quantities of GLMs can also approximates to

$$
\begin{array}{r}
y_{i}^{[n-1]}=\sum_{k=0}^{p} \alpha_{i k} y^{(k)}\left(x_{n-1}\right) h^{k}+O\left(h^{p+1}\right), \\
y_{i}^{[n]}=\sum_{k=0}^{p} \alpha_{i k} y^{(k)}\left(x_{n}\right) h^{k}+O\left(h^{p+1}\right) .
\end{array}
$$

The subclass of GLMs which is DIMSIMs has the leading coeffcient matrix, $A$, that can have four different structures known as types, depending on whether the intended application is non-stiff or stiff and on whether the computer architecture is sequential or parallel [2]. DIMSIMs can be divided into four types such as follows:

- Type I.

The coefficient matrix $A$ of this type considered as lower triangular matrix and its diagonals equal to zero. The type one required to solve the non-stiff problem on a sequential computer.

- Type II.

The coefficient matrix $A$ of this type considered as lower triangular matrix and its diagonals equal to constant. The type two required to solve the stiff problem on a sequential computer.

- Type III.

The coefficient matrix $A$ given for this type as zero matrix. This type required for non-stiff problem in a parallel environment.

- Type IV.

The coefficient matrix $A$ is assumed in this type as diagonal matrix. This type required for stiff problem in a parallel environment.

The class of GLMs include many special methods, as given in [2] and [3]. In particular, these methods have subclass of DIMSIMs considered in [1] and further investigated in [4], [5] and [6], two-step RK methods considered in [7] and further investigated in [8] and [9]. The DIMSIMs particularly had been investigated by Will Wright in his $\mathrm{PhD}$ thesis [10]. DIMSIMs have also been discussed in a monograph given by Jackiewicz [11], Hairer and Wanner [12] and a review paper by Butcher [13]. 
In the recent years, it has been challenging to construct an efficient methods that are more accurate and more efficient than original methods. In [14], he described the construction of algebraically stable DIMSIMs by introducing a new idea such as $\epsilon$-algebraic stability and study its consequences. In [15], they introduced a new class of implicit-explicit DIMSIMs, where the non-stiff part is treated by an explicit formula and the stiff part is treated by an implicit formula. In the same year, Famelis and Jackiewicz [16] gave the new construction of DIMSIMs based on Differential Evolution. In this paper, the advantages of using the extrapolation technique with explicit DIMSIMs methods are given for some non-stiff problems.

\section{Order Conditions}

The order conditions of DIMSIMs can be considered by using the steps between $x_{n-1}$ and $x_{n}$ with step-size $h$. The output approximations of DIMSIMs is assumed to have the same order. The order $p$ and stage order $q$ are considered to be the same.

Recall the form of GLMs for a single step

$$
\begin{aligned}
& Y^{[n]}=\operatorname{Ahf}\left(Y^{[n]}\right)+U y^{[n-1]} \\
& y^{[n]}=\operatorname{Bhf}\left(Y^{[n]}\right)+V y^{[n-1]} .
\end{aligned}
$$

In order to satisfy the order condition of DIMSIMs, it is necessary to consider the following

$$
\begin{aligned}
\exp (c z) & =z A \exp (c z)+U Z+O\left(h^{p+1}\right) \\
\exp (z) Z & =z B \exp (c z)+V Z+O\left(h^{p+1}\right)
\end{aligned}
$$

where

$$
\exp (c z)=\left[\begin{array}{c}
\exp \left(c_{1} z\right) \\
\exp \left(c_{2} z\right) \\
\vdots \\
\exp \left(c_{s} z\right)
\end{array}\right], \quad Z=\left[\begin{array}{c}
1 \\
z \\
\vdots \\
z^{p} \cdot
\end{array}\right]
$$

\section{Construction of Type I DIMSIMs}

Explicit solvers have updated the type I DIMSIMs which has been considered firstly by [1]. The methods considered are with conditions $p=q=r=s$. The stability function during these methods considered by

$$
p(\omega, z)=\omega^{s-1}(\omega-R(z)),
$$

where

$$
R(z)=1+z+\frac{z^{2}}{2 !}+\cdots+\frac{z^{s}}{s !},
$$

which is approximate to the exponential function $\exp (z)$. This methods have similar stability as the RK methods of order $s$. By following [4], the stability function is given by

$$
p(\omega, z)=\omega^{s}-p_{1}(z) \omega^{s-1}+\cdots+(-1)^{s-1} p_{s-1}(z) \omega+(-1)^{s} p_{s}(z) \omega
$$


where

$$
\begin{aligned}
p_{1}(z) & =1+p_{11} z+p_{12} z^{2}+\cdots+p_{1 s} z^{s}, \\
p_{2}(z) & =p_{21} z+p_{22} z^{2}+\cdots+p_{2 s} z^{s}, \\
\vdots & \\
p_{s-1}(z) & =p_{s-1, s-2} z^{s-2}+p_{s-1, s-1} z^{s-1}+p_{s-1, s} z^{s}, \\
p_{s}(z) & =p_{s, s-1} z^{s-1}+p_{s s} z^{s},
\end{aligned}
$$

where $p_{i, j}$ are coefficients of polynomial $p_{i}(z)$ relying on $a_{i j}, i=$ $1,2, \cdots, s, j=1,2, \cdots, i-1$ and $v_{i}, i=1,2, \cdots,, s-1$. By solving the form of $(s-1)(s+2) / 2$ for non-linear problems

$$
p_{k l}=0, \quad k=2,3, \cdots, s, \quad l=k-1, k, \cdots, s,
$$

with respect to the value $(s-1)(s+2) / 2$. The coefficient matrix $B$ can be obtained by using the following condition:

$$
B=B_{0}-A B_{1}-V B_{2}+V A,
$$

The coefficients $p_{i j}$ on the other hand can be computed by using the Fourier series approach.

A Fourier series approach can be summarized as follows. Consider that $w_{\mu} \mu=1,2, \ldots, N_{1}$, are complex numbers spanned uniformly on the unit circle, where $N_{1}$ denotes a sufficiently large integer. Multiplying the following relation

$$
p\left(w_{\mu}, z\right)=w_{\mu}^{s}-p_{1}(z) w_{\mu}^{s-1}+\cdots+(-1)^{s-1} p_{s-1}(z) w_{\mu}+(-1)^{s} p_{s}(z)
$$

by $\omega_{\mu}^{k-s}, k=1,2, \cdots, s$, and using the summation with respect to $\mu$, then

$$
(-1)^{k} p_{k}(z)=\frac{1}{N_{1}} \sum_{\mu=1}^{N 1} \omega_{\mu}^{k-s} p\left(\omega_{\mu}, z\right) .
$$

In the same way, multiplying

$$
p_{k}\left(z_{v}\right)=p_{k, k-1} z_{v}^{k-1}+p_{k k} z_{v}^{k}+\cdots+p_{k s} z_{v}^{s}, \quad k=2,3, \cdots, s,
$$

by $z_{v}^{-1}$, and using the summation with respect to $v$, then

$$
\begin{aligned}
p_{k l} & =\frac{1}{N 2} \sum_{v=1}^{N_{2}} z_{v}^{-1} p_{k}\left(z_{v}\right), \\
& =(-1)^{k} \frac{1}{N_{1} N_{2}} \sum_{\mu=1}^{N_{1}} \sum_{v=1}^{N_{2}} \omega_{\mu}^{k-s} z_{v}^{-1} p\left(\omega_{\mu, z_{v}}\right) .
\end{aligned}
$$

This system can be numerically solved as

$$
\sum_{\mu=1}^{N_{1}} \sum_{v=1}^{N_{2}} \omega_{\mu}^{k-s} z_{v}^{-1} p\left(\omega_{\mu, z_{v}}\right)=0,
$$

Therefore, the polynomial of type I DIMSIMs can be obtained from the following equation

$$
p(\omega, z)=\operatorname{det}(Q(\omega, z)),
$$

where

$$
Q(\omega, z)=\omega(I-z A)-V-z B_{0}+z A B_{1}+z V B_{2} .
$$

and the determinant det is a scalar value that can be computed from the elements of a square matrix and encodes certain properties of the linear transformation. 


\section{Extrapolation Technique}

Extrapolation technique is a powerful computational technique that can be successfully applied in the efforts to improve the accuracy of the approximate solutions in solving ordinary differential equations. Extrapolation was first been introduced by Richardson in 1911. It is an approximation method in the numerical solution of differential equations. Richardson showed that the approximations arising from finite difference scheme can be expressed in the form of

$$
T(h)=\tau_{0}+\tau_{2} h^{2}+\tau_{4} h^{4}+\ldots+O\left(h^{8}\right),
$$

where $h$ is stepsize, $T(0)=\tau_{0}$ is an adjustable parameter and $\tau_{2}, \tau_{4}$ are independent of $h$.

The extrapolation formula by the Aitken-Neville formula is given by

$$
T_{i j}=T_{i, j-1}+\frac{T_{i, j-1}-T_{i-1, j-1}}{\left(\frac{m_{1}}{m_{i-j+1}}\right)-1}, \quad m=1,2, \ldots
$$

where $i=j=2, \ldots, n$.

There are two modes of extrapolation that can be applied in numerical approach. They are passive and active extrapolations. When extrapolated values are not used in any subsequent computation of other extrapolated values, the process is called passive extrapolation whereas if the extrapolated values are used at the end of a step as the starting value for the next step, it is called active extrapolation. Since the extrapolation can increase the accuracy and efficiency of the approximate solutions, many researchers during these recent years are still finding and developing the best ways to use this technique. Gorgey [17] showed that passive extrapolation by the two stage Gauss method is more efficient than the active extrapolation in solving the linear and nonlinear stiff problems. Besides, the paper in [18] expressed the computing time spent by the Richardson extrapolation for both types active and passive is more than ten times smaller than the corresponding computing time by the Backward Euler Formula. By following the research by Mona, Lagzi and Havasi as given in [18], it is concluded that extrapolation is a powerful tool for increasing the accuracy and efficiency with regard to the computational cost especially when the accuracy requirement is not extremely low. In [19], it is shown that a new procedure to build stabilized explicit RK methods based on Richardson extrapolation with higher order is studied. The strategy was easy and allows deriving stabilized explicit RK methods with order as high as desired. However, for low order, the stability property were a little shorter than other methods without this technique.

In [20], the advantages of using extrapolation are given as follows:

- It is possible to use the extrapolation technique in order to improve the accuracy of the numerical experiments.

- The active type of extrapolation technique combining with Trapezoidal role can make the computations becomes unstable.

- The computational cost of extrapolation technique is less than the underlying numerical methods for prescribing the accuracy.

- The application with applying the Backward Euler formula with active active type of extrapolation is leading to a stable procedure. 
In this paper, passive extrapolation technique is applied to improve the accuracy of the approximate solutions by the explicit DIMSIMs methods of higher order in solving non-stiff problems. In the next section, we will consider the numerical experiments of explicit DIMSIMs with extrapolation technique.

\section{Numerical Results}

In this section, we discuss the results of numerical experiments obtained by the MATLAB code dim18-extrap.m. This code is based on explicit DIMSIMs with extrapolation technique of higher order $p_{\max }=8$. . To compare, we also present the results obtained by the MATLAB code dim18.m. This code is based on explicit DIMSIM$\mathrm{s}$ without extrapolation of high order $p_{\max }=8$, as being described in [21].

The decision about determining the new order within $1 \leq p \leq 8$ relaying on the following form

$$
\text { ratio }=\frac{\left\|\operatorname{est}\left(x_{n}, p_{n}\right)\right\|}{\left\|\operatorname{est}\left(x_{n}, p_{n}-1\right)\right\|}
$$

where

$$
\operatorname{est}\left(x_{n}, p_{n}-1\right)=C\left(p_{n}-1\right) z^{[n]}\left(p_{n} m+1:\left(p_{n}+1\right) m\right)
$$

Now if the previous step was not rejected and ratio $<r_{\min }, \quad p_{n}<$ $p_{\max }$, the new order will be choose as $p_{n+1}=p_{n}+1$. Besides, if the ratio $>r_{\max }$ and $p_{n}>1$, then the new order in this case leads to $p_{n+1}=p_{n}-1$. By contract with this case, the order will be not changed. During the above codes dim18-extrap.m and dim18.m, the parameters chosen as $r_{\min }=0.9, r_{\max }=1.1$ and the maximal orer is $p_{\max }=8$.

The results will show which one of these methods is efficient in solving non-stiff problems such as Van der Pol (VDP) and Brusselator (BRUS) problems. The first test problem is Van der Pol (VDP) which is defined as follows:

$$
\begin{aligned}
& y_{1}^{\prime}=y_{2}, \\
& y_{2}^{\prime}=\left(\left(1-y 1^{2}\right) y_{2}-y_{1}\right) / \epsilon,
\end{aligned}
$$

where the first equation denotes the non-stiff equation while the second equation denotes stiff equation with including small $\epsilon$. In order to solve the non-stiff problem, then $\epsilon$ considered here is equal to one. The initial values of VDP are given as follows

$$
\begin{aligned}
& y_{1}(0)=2, \\
& y_{2}(0)=-\frac{2}{3}+\frac{10}{81} \epsilon-\frac{292}{2187} \epsilon^{2}-\frac{1814}{19683} \epsilon^{3}+O\left(\epsilon^{4}\right) .
\end{aligned}
$$

The problem is integrated to $x_{n}=3$.

The second test problem is Brusselator (BRUS) which is an auto catalytic oscillating chemical reaction problem. It is a system of two ordinary differential equations that are given by

$$
\begin{aligned}
& y_{1}^{\prime}=1+y_{1}^{2} y_{2}-4 y_{1}, \\
& y_{2}^{\prime}=3 y_{1}-y_{1}^{2} y_{2} .
\end{aligned}
$$

where $y_{1}(0)=1.5$ and $y_{2}(0)=3$. The problem is integrated to $x_{n}=1$ by using $h=0.05$. 
For the two problems, four figures are given. These figures give the numerical results of stepsize and CPU-time versus global errors for order 8 DIMSIMs with and without extrapolation technique. All the numerical computational are performed by using MATLAB. The CPU-time is measured by applying tic and toc as given in MATLAB. The starting absolute and relative tolerances are $t o l=10^{7}$.

Figure 1 gives the numerical results for VDP test problem. From the figure, we can see the extrapolation technique with explicit DIMSIMs of order- 8 gives better efficiency than explicit DIMSIMs without extrapolation. Furthermore, the numerical results given in Figure 2 show that the extrapolation technique with explicit DIMSIMs of order- 8 also gives greater efficiency than explicit DIMSIMs without extrapolation for BRUSS test problem.
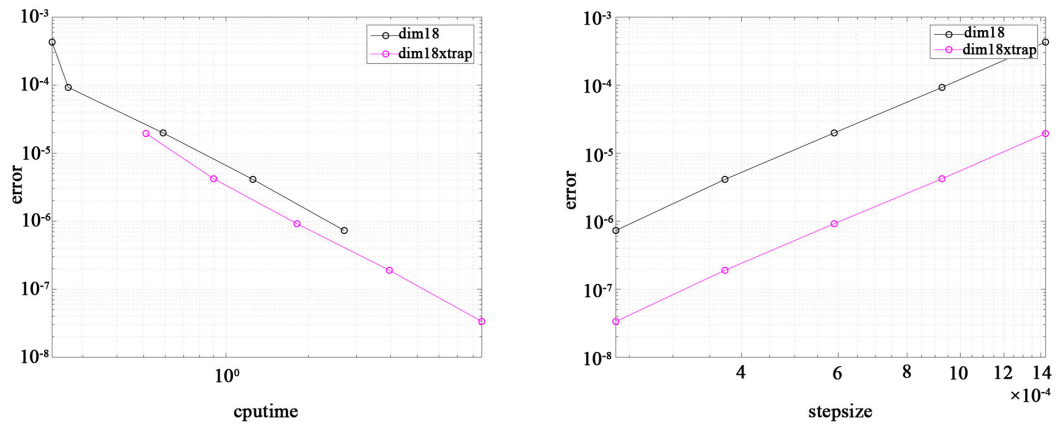

Figure 1. Numerical results for VDP problem by order-8 DIMSIM with and without extrapolation.
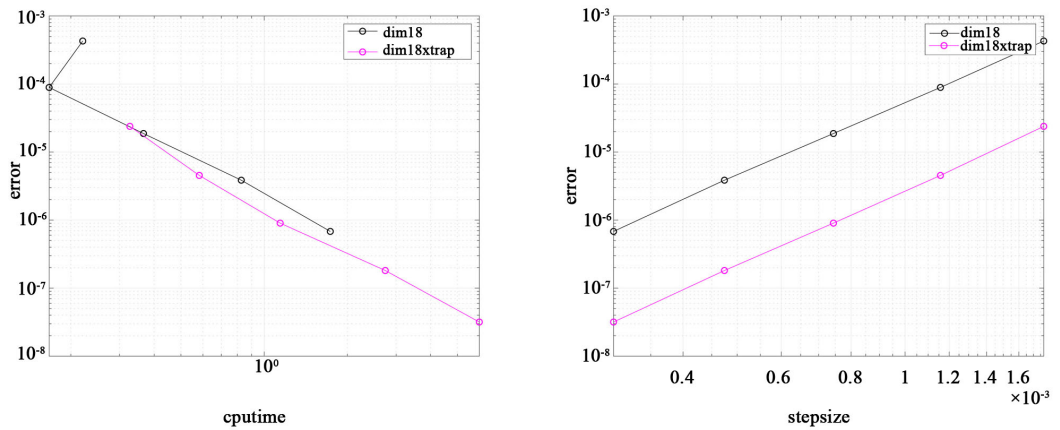

Figure 2. Numerical results for BRUS problem by order-8 DIMSIMs with and without extrapolation.

\section{Conclusions}

In this paper, we discuss some issues related the development of explicit DIMSIMs for the numerical solution of non-stiff differential equations. This paper also gives the application of passive extrapolation by the order-8 explicit DIMSIMs. As we can see from numerical results, the extrapolation technique with explicit DIMSIMs of order-8 gives better efficiency than explicit DIMSIMs without extrapolation in solving VDP and BRUS test problems.

Future work will involve verifying the efficiency of implicit DIMSIMs of higher order with extrapolation in solving stiff differential equations. 


\section{Acknowledgements}

The authors would like to extend their gratitude to the Ministry of Higher Education in Malaysia for providing the research grant FRGS, Vote No: 2015-0158-104-02.

\section{Conflicts of Interest}

The authors declare no conflicts of interest regarding the publication of this paper.

\section{References}

[1] Butcher, J.C. (1993) Diagonally-Implicit Multi-Stage Integration Methods. Applied Numerical Mathematics, 11, 347-363. https://doi.org/10.1016/0168-9274(93)90059-Z

[2] Jackiewicz, J. (2009) General Linear Methods for Ordinary Differential Equations. John Wiley \& Sons, Hoboken, NJ. https://doi.org/10.1002/9780470522165

[3] Butcher, J.C. (1987) The Numerical Analysis of Ordinary Differential Equations: Runge-Kutta and General Linear Methods. John Wiley \& Sons, Chichester, New York.

[4] Butcher, J.C. and Jackiewicz J. (1998) Construction of High Order Diagonally Implicit Multistage Integration Methods for Ordinary-Differential Equations. Applied Numerical Mathematics, 27, 1-12. https://doi.org/10.1016/S0168-9274(97)00109-8

[5] Butcher, J.C. and Jackiewicz, J. (1996) Construction of Diagonally Implicit General Linear Methods of Type 1 and 2 for Ordinary Differential Equations. Applied Numerical Mathematics, 21, 385415. https://doi.org/10.1016/S0168-9274(96)00043-8

[6] Butcher, J.C., Chartier, P. and Jackiewicz, J. (1997) Nordsieck Representation of DIMSIMs. Numerical Algorithms, 16, 209-230. https://doi.org/10.1023/A:1019195215402

[7] Jackiewicz, Z. and Tracogna, S. (1995) A General Class of TwoStep Runge-Kutta Methods for Ordinary Differential Equations. SIAM Journal on Numerical Analysis, 32, 1390-1427. https://doi.org/10.1137/0732064

[8] Cholloma, J. and Jackiewicz, Z. (2003) Construction of Two-Step Runge-Kutta Methods with Large Regions of Absolute Stability. Journal of Computational and Applied Mathematics, 157, 125137. https://doi.org/10.1016/S0377-0427(03)00382-0

[9] Conte, D., D'Ambrosio, R. and Jackiewicz, Z. (2010) Two-Step Runge-Kutta Methods with Quadratic Stability Functions. Journal of Scientific Computing, 44, 191-218.

https://doi.org/10.1007/s10915-010-9378-x

[10] Wright, W. (2002) General Linear Methods with Inherent RungeKutta Stability. Ph.D. Thesis, The University of Auckland, Auckland, New Zealand. 
[11] Jackiewicz, Z. (2005) Construction and Implementation of General Linear Methods for Ordinary Differential Equations: A Review. Journal of Scientific Computing, 25, 29-49. https://doi.org/10.1007/s10915-004-4631-9

[12] Hairer, E. and Wanner, G. (1996) Solving Ordinary Differential Equations II. Stiff and Differential-Algebraic Problems. Springer, Berlin. https://doi.org/10.1007/978-3-642-05221-7

[13] Butcher, J.C. (2006) General Linear Methods. Acta Numerica, 15, 157-256. https://doi.org/10.1017/S0962492906220014

[14] Izzo, G. and Jackiewicz, Z. (2014) Construction of Algebraically Stable DIMSIMs. Journal of Computational and Applied Mathematics, 261, 72-84. https://doi.org/10.1016/j.cam.2013.10.037

[15] Jackiewicz, Z. and Mittelmann, H. (2017) Construction of IMEX DIMSIMs of High Order and Stage Order. Applied Numerical Mathematics, 121, 234-248.

https://doi.org/10.1016/j.apnum.2017.07.004

[16] Famelis, I.T. and Jackiewicz, Z. (2017) A New Approach to the Construction of DIMSIMs of High Order and Stage Order. $A p$ plied Numerical Mathematics, 119, 79-93.

https://doi.org/10.1016/j.apnum.2017.03.015

[17] Gorgey, A. (2012) Extrapolation of Symmetrized Runge-Kutta Methods. Ph.D. Thesis, The University of Auckland, New Zealand.

[18] Mona, T., Lagzi, I. and Havasi, A. (2015) Solving ReactionDiffusion and Advection Problems with Richardson Extrapolation. Journal of Chemistry, 2015, Article ID: 350362. https://doi.org/10.1155/2015/350362

[19] Martín-Vaquero, J. and Kleefeld, B. (2016) Extrapolated Stabilized Explicit Runge-Kutta Methods. Journal of Computational Physics, 326, 141-155. https://doi.org/10.1016/j.jcp.2016.08.042

[20] Farago, I., Havasi, A. and Zlatev, Z. (2010) Efficient Implementation of Stable Richardson Extrapolation Algorithms. Computers and Mathematics with Applications, 60, 2309-2325.

https://doi.org/10.1016/j.camwa.2010.08.025

[21] Butcher, J.C., Chartier, P. and Jackiewicz, Z. (1999) Experiments with a Variable-Order Type 1 DIMSIM Code. Numerical Algorithms, 22, 237-261. https://doi.org/10.1023/A:1019135630307 